\title{
Towards Ultra-Reliable Low-Latency Underwater Optical Wireless Communications
}

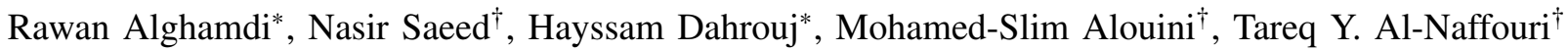 \\ *Department of Electrical and Computer Engineering, Effat University, Jeddah, 22332, Saudi Arabia. \\ ${ }^{\dagger}$ Computer, Electrical, and Mathematical Sciences \& Engineering (CEMSE) Division, \\ King Abdullah University of Science and Technology (KAUST), Thuwal, 23955-6900, Saudi Arabia.
}

\begin{abstract}
The superiority of optical communications in underwater mediums, in terms of higher data rate and reliability, makes underwater optical wireless communications (UOWC) more favorable to provide ultra-reliable low-latency underwater communications, as compared to other wireless technologies, e.g., acoustic and radio frequency (RF) communications. UOWC limited transmission range, however, remains a major hurdle against assessing its true deployment benefits, which motivates for the necessity of developing practical routing protocols for multihop underwater optical wireless sensor networks (UOWSNs). This paper sheds light on the existing state-of-art UOWC routing protocols, the majority of which requires centralized implementation with large end-to-end delay. The article further proposes routing algorithms which can be implemented in a distributed fashion across the multi-hop links, with a reasonable amount of information exchange. The merits of the proposed algorithms are particularly highlighted through illustrative simulations, which show how the proposed strategies outperform the classical protocols, both in terms of reliability and end-to-end latency. Finally, the paper shows how the proposed distributive routing protocols achieve ultra-reliable low-latency underwater communications.

Index Terms-Underwater optical wireless communications, underwater optical wireless sensor network, routing protocols, distributed routing protocols, Ultra-reliable low-latency communication.
\end{abstract}

\section{INTRODUCTION}

Oceans, which occupy $97 \%$ of the planet Earth surface, produce numerous benefits to humankind, e.g., food supply, climate regulation, recreation, transportation, and medicine. It is reported in [2] that businesses based on oceans contribute more than 500 billion US dollars to the world economy. Nevertheless, $95 \%$ of oceans remain unexplored, which necessitates deploying underwater wireless sensor networks (UWSNs), as a means to explore oceans and reserve their bio-diversity.

UWSNs have the valuable capabilities of enhancing diverse applications such as offshore and ocean sampling, ocean exploration, navigation, recreation, surveillance, and disaster management. Such applications are best enabled through the proper choice of the underwater wireless communication technology, which would provide the high data rate and low latency metrics required to facilitate reliable broadband services. The

A detailed technical report on this paper can be found in [1]. Hayssam Dahrouj would like to thank Effat University in Jeddah, Saudi Arabia, for funding the research reported in this paper through the Research and Consultancy Institute. The work is also supported by the Office of Sponsored Research (OSR) at King Abdullah University of Science and Technology (KAUST). nature of the underwater environments, however, enforces severe challenges on the establishment of a real-time broadband wireless communication link. Acoustic waves, which are currently available for military and commercial purposes, support long communication ranges in aquatic mediums up to tens of $\mathrm{Kms}$, with a restrained data rate performance and high latency induced by the low propagation speed [3]. Similarly, the electromagnetic RF waves are highly attenuative for underwater communications and bounded by the extremely low-frequency band, which imposes utilizing large antenna sizes. RF-based underwater communication systems, thus, do not provide high data rate services in practical UWSNs [4].

Underwater optical wireless communication (UOWC) is an alternative approach to acoustic and RF for creating a reliable, real-time broadband link. Optical waves distinguishable feature is their high propagation speed, which substantially reduces the transmission delay and supports broadband communication, even in the presence of high absorption and scattering [3]. The practical deployment of UOWC, however, suffers from the short communication range, which is limited to few tens of meters in aquatic mediums, due to the physical properties of underwater propagation and intrinsic properties of light. Despite such limitations, the visible spectrum offers a unique behavior of absorption and scattering in the blue/green region [5]. Utilizing a wavelength within such region, therefore, guarantees low latency underwater wireless communication for relatively long ranges.

A wireless sensor network (WSN), in which multiple sensors communicate using UOWC technology, is referred to as underwater optical wireless sensor network (UOWSN) in the context of this paper. Given the transmission range of UOWC, the real assessment of UOWSN stipulates developing a multihop network where the sensed information is transmitted over a multi-hop path or a series of relaying nodes. This multi-hop implementation has the advantages of increasing the network lifetime and coverage, extending the communication range, and reducing the interference level [6], mainly when a proper routing protocol governs the way the information travel among the many sensors. It becomes, therefore, essential to develop practical routing protocols for underwater multihop networks, as a means to achieve reliable and efficient communication links, in an attempt to realize ultra-reliable low latency underwater wireless communication systems. A routing protocol which aims to support an ultra-reliable low- 
latency underwater communication (URLLUC) in UOWSNs must achieve a minimum end-to-end (E2E) latency of $1 \mathrm{~ms}$ and maintain a communication link reliability with bit error rate between $\left[10^{-6}-10^{-9}\right]$ [7], [8]. This paper considers a multi-hop UOWSN system and addresses the routing problem to achieve an ultra-reliable low-latency underwater communication. The paper main contributions are routing algorithms which can be implemented in a distributed fashion across the multi-hop links, with a reasonable amount of information exchange. The simulation results show how the proposed algorithms outperform the classical protocols, both in terms of reliability and end-to-end latency.

\section{STATE-OF-THE-ART}

From a system design perspective, routing protocols are the policies that determine the optimized paths along which information travel based on system design requirements [5]. The existing work on routing protocols can be classified into two categories: centralized and distributed. The literature on centralized routing protocols is particularly rich and addresses various network environments, such as terrestrial, underground and underwater networks, e.g., see [5] and references therein. Centralized routing protocols commonly utilize a central node, which is responsible for collecting all the information from the network, to estimate the optimal path between the source and the target, under a given performance metric constraint [5]. For large-scale sensor networks, however, centralized implementation of a routing algorithm may become infeasible, especially in underwater scenarios, due to the absence of centralized computing processors.

On the other hand, distributed routing adopts a more practical approach, as the source node decides on the forwarding node using the local information available only [9]. Towards that end, references [10], [11] propose distinct routing protocols for underwater acoustic wireless sensor networks (UAWSNs). Such routing protocols are, however, not suitable for UOWSNs, as they do not consider the physical characteristics and probabilistic models of light propagation in the aquatic medium. Also, the routing protocols for UAWSNs consider longe-range omnidirectional communication, which is not valid for UOWSNs because of its directive nature and limited communication range.

To the best of the authors' knowledge, the recent work in [12] is the first to propose a new, albeit centralized, routing protocol in UOWSNs, by using a Dijkstra's algorithm-based approach [13]. Our current paper, therefore, aims at investigating different routing protocols in UOWSNs and proposes new algorithms which are amenable to distributed implementation. The paper first examines the characteristics of UOWC in the line of sight (LoS) condition with different types of water and different beam-widths of the optical signal. In summary, here are the contributions of the paper:

- Examining the characteristics of UOWC in the line-ofsight (LoS) condition with different types of seawater and different beam-widths of the optical signal.
- Investigating centralized routing protocols [12], and proposing new distributed routing protocols.

- Evaluating each protocol in terms of the design parameters of URLLUC, i.e., end-to-end bit error rate (BER) and end-to-end time delay.

The remainder of the article is organized as follows. Section III examines the characteristics of UOWC in LOS conditions under different propagation characteristics. Section IV investigates the most recent routing protocols, proposes new distributed algorithms, and evaluates their respective performance. Final remarks are concluded in section VI.

\section{UNDERWATER OPTICAL WIRELESS COMMUNICATION}

This section paves the way for the routing protocols described in section IV, as it examines the propagation characteristics and limitations of optical waves in underwater mediums. This section further presents the architecture of the UOWSN and highlights the channel budget link and its associated BER.

\section{A. Underwater Propagation Characteristics of Optical Waves}

Optical waves are a promising transmission carrier for enabling URLLUC, due to their ability to achieve broadband links with high data rate (Gbps) and low latency [5]. They, however, suffer from several impairments caused by their inherent and apparent properties such as absorption, scattering, salinity, temperature, and misalignment of the transceivers due to the sea surface movements [5]. Reference [5] particularly highlights the impact of inherent and apparent properties of light on modeling the UOWC channel, which explains why it is critical to consider the physical layer characteristics and limitations of the UOWC systems while designing routing protocols in underwater mediums. In the following, first, we define the link budget for UOWC, then evaluate and show the simulations of a single-hop BER in LoS conditions. Finally, we extend the scenario of single hop BER calculation to the multi-hop case.

1) UOWC Link Budget: In underwater mediums, the optical waves experience a combination of absorption and scattering effects, which are characterized by Beer-Lambert's law, and which cause extinction coefficients that depend on the water type and the utilized wavelength [3]. Not only do light waves get attenuated by absorption and scattering effects, but they also experience energy loss when they spread larger than the receiver aperture. The overall path loss, therefore, can be expressed as

$$
P L=\frac{A \cos (\theta)}{2 \pi\left(1-\cos \left(\theta_{0}\right)\right) d^{2}} \exp \left(\frac{-t(\lambda) d}{\cos (\theta)}\right),
$$

where $t(\lambda)=a(\lambda)+s(\lambda)$ is the extinction coefficient for absorption and scattering at a wavelength of $\lambda$ [5]. $\theta$ is the angle between the transmitter-receiver trajectory, $A$ is the aperture area of the receiver, $d$ is the Euclidean distance between the transmitter and the receiver, and $\theta_{0}$ is the laser beam divergence angle. The characterization of path loss in (1) establishes an exponential relationship between the received optical power and the variety of water types (clear ocean, 


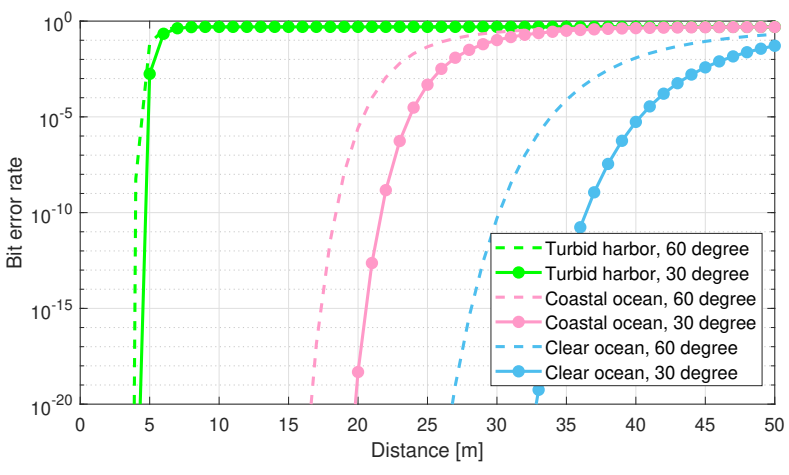

Fig. 1. Bit error rate as a function of the distance between the transmitter and the receiver for various water types and laser beam angles for a UOWC channel in LoS conditions.

coastal ocean, and turbid harbor). The received optical power for a LoS link is described as follows [12]

$$
P_{R_{x} l o s}=P_{T x} \eta_{T x} \eta_{R x} \times P L,
$$

where $P_{T x}$ is the average optical transmitter power, $\eta_{T}$ and $\eta_{R}$ are the optical efficiencies of the transmitter and the receiver, respectively. It is shown in our detailed technical report that the received power is high for the low values of extinction coefficients [1]. Furthermore, narrowing the laser beam-width results in increasing the received signal power, as the light beam concentrates on the receiver aperture [1].

2) Bit Error Rate: In the existing literature, researchers commonly utilize On-Off-Keying (OOK) modulation technique with direct detection (DD) method for optical wireless communication. In [3], the authors express bit error rate (BER) as a function of the number of photons arrival during a pulse duration of $T$. When a large number of photons are transmitted, the photon arrival rate is a random process modeled as a Poisson distribution which, according to the central limit theorem, can approximate the BER by a Gaussian distribution as

$$
P(e)=\frac{\operatorname{erfc}\left[\sqrt{\frac{T}{2}}\left(\sqrt{r_{1}}-\sqrt{r_{0}}\right)\right]}{2},
$$

where $\operatorname{erfc}(\cdot)$ is the complementary error function, $r_{0}$ and $r_{1}$ are the numbers of photon arrivals when transmitting a binary bit 0 and a binary bit 1 , respectively. The numbers of photon arrivals for $r_{1}$ and $r_{0}$ are given by

$$
r_{0}=r_{d c}+r_{b g}, r_{1}=r_{d c}+r_{b g}+r_{p},
$$

where $r_{d c}$ and $r_{b g}$ are the source of additive noise due to dark counts and background illumination, respectively. In (4) $r_{p}=\frac{P_{R_{x}} \eta \lambda}{T R h c}$, is the photon arrival rate for a given data rate $R$, where $\eta$ is the detector counting efficiency, $h$ is Plank's constant and $c$ the speed of light in the water $\left(2.25 \times 10^{8} \mathrm{~m} / \mathrm{s}\right)$, respectively [14].

Fig. 1 illustrates the BER performance based on the analytical expression in [3] for a single link in different types of water (clear ocean, coastal ocean, and turbid harbor) and with different laser beam-width angles [15]. Fig. 1 shows that the BER performance is high for low extinction coefficient case (clear ocean water) and with the narrowed beam-width angle, as the received signal power is higher in such cases. Fig. 1 also shows that the turbid harbor water, which has the highest extinction coefficient, provides the worst BER for all beam-width values even at very short distances.

End-to-end Bit Error Rate: The end-to-end BER utilized for multi-hop networks is calculated using the recursive joint probability. Assuming that the channels of successive hops are statistically independent, the end-to-end BER is given as [6]

$$
P_{Z}(e 2 e)=\left(1-P_{Z-1}\right) P(Z)+(1-P(Z)) P_{Z-1} \text {. }
$$

Here, $P(i)$ is the single link BER from node $i-1$ to node $i$, $P_{i}$ is the end-to-end BER from source node to node $i$, and $i=\{1,2,3, \ldots, Z\}$. Therefore, $\left(1-P_{i}\right)$ is the probability of a bit transmitted correctly from a source node to the $i$-th node and $(1-P(i))$ is the probability of a correct bit transmission in the single link between node $i-1$ and node $i$.

3) Maximum Transmission Range: Towards keeping a reliable connected network, we impose a constraint on the achievable transmission range. We can derive an analytical expression of the latter by defining a BER threshold $\mathrm{BER}_{t h}$, substituting the values of $P_{R_{x} l o s}$ and $r_{p}$ in (3), and solving it for the transmission range yields (6). Note that, in (6), one can obtain a BER ${ }_{t h}$ between any two connected nodes if their distance is within the maximum allowable transmission range $R_{\max }$, where $W_{0}(\cdot)$ denotes the principal real-valued branch of the Lambert function.

\section{ROUTING PROTOCOLS FOR UOWSNS}

As illustrated in the introduction section, routing plays a significant role in sustaining the transmission path in a communication system. The various routing protocols maintain and advocate distinct channel characteristics. The optimization of routing protocols alters with different applications and evaluation metrics such as end-to-end BER, delay efficiency, energy efficiency, and complexity. In the context of this paper, we define a possible route as the route connecting the source and the target without visiting any vertex (node) more than once. In this section, we first define the network graph model and then revisit the centralized routing protocol proposed in [12] and propose two novel distributed routing protocols. Finally, we assess the performance of all the three routing protocols through extensive numerical results in terms of endto-end reliability and delay.

\section{A. Network Graph Model}

In the context of this paper, we consider a UOWSN as a digraph $G=(V, E)$, where $V$ is the set of vertices/sensor nodes, $M$ is the set of sensor nodes which are either deployed at the seabed or moored, and $B$ is the set of surface buoys, i.e., $V=M+B$ and $E$ is the set of directed edges with weights of their Euclidean distances. Fig. 2 illustrates an example of the UOWSN under consideration. The sensor nodes are responsible for monitoring its surroundings, and send the collected information to the surface buoys through UOWC links in a multi-hop fashion. An edge $e_{i j}$ exists between node $i$ 


$$
R_{\max }=\left(\frac{2 \cos (\theta)}{t(\lambda)}\right) W_{0}\left[\frac{t(\lambda)}{2 \cos (\theta)} \frac{1}{\sqrt{\frac{2 \pi\left(1-\cos \left(\theta_{0}\right)\right) T R h c}{P_{T x} \eta_{T x} \eta_{R x} \eta \lambda A \cos (\theta)}\left[\left(\sqrt{r_{0}}+\sqrt{\frac{2}{T}} \operatorname{erfc}^{-1}\left(2 \mathrm{BER}_{t h}\right)\right)^{2}-r_{0}\right]}}\right] .
$$

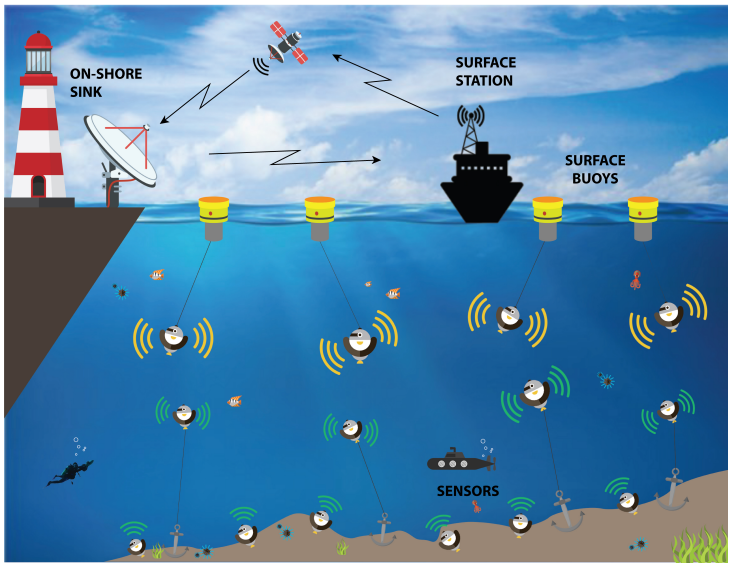

Fig. 2. An architecture of underwater optical wireless sensor network.

and $j$, if and only if the single link BER of that edge is within the predefined $\mathrm{BER}_{t h}=10^{-9}$. When a forwarding node has an empty set of candidates, the forwarding node may increment its $\mathrm{BER}_{t h}$ repeatedly to establish a successful transmission. A successful data transmission, in this case, is defined as the multi-hop path from the source node continuously to the target node, without visiting any vertex/edge more than once. Above that, we introduce an additional condition where a node $i$ tactfully sectors the network to properly route the information from the source to the target with an angle which is equal to the divergence angle $\theta_{0}$ directed at the final target. That is, for an edge $e_{i j}$ to have a real value (exists), node $j$ must fall within the optical sector from $i$ to the target node. This strategy is more practical for UOWSNs, as the underwater optical transmission is directive and it restricts the routing protocols from forwarding to nodes which are not positioned towards the target as to avoid having the end-to-end path with higher latency and signal attenuation.

\section{B. Centralized Routing Protocol (CRP)}

Recently, the authors in [12] propose a centralized routing protocol (CRP) which utilizes Dijkstra's shortest path algorithm to route data packets in a multi-hop fashion. Following the same directed graph defined in the previous section, the routing protocol selects the shortest distance path from the source node to the target node. The main issue in implementing the CRP proposed in [12] is that it necessitates the presence of a centralized computing processor, which is not possible to deploy in practice in underwater mediums. Furthermore, CRP computes the best possible path in terms of distance; however, it cannot choose the best path in terms of both distance and

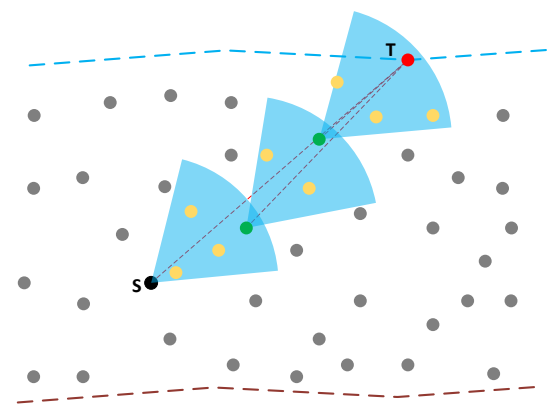

Fig. 3. Path selection process for a distributed sector-based routing protocol, where the blue sector covers only the allowable transmission area and the green dots are the forwarding nodes, the yellow ones are marking the rest of the possible candidates and the red dashed line is an imaginary line marking how the divergence angle is bisected with respect to the target.

number of edges, which results in a substantial end-to-end delay, as illustrated later in section $\mathrm{V}$.

\section{Distributed Sector-based (DS) Routing Protocol}

To resolve the above issues of CRP, we propose a distributed sector-based routing protocol (DS), which can be seen as a combination of the nearest neighbor problem [16] and the traveler salesman problem [13], i.e., a special greedy version of the CRP. The distributed nature of DS exists in the fact that each vertex/node is only aware of its neighboring nodes. In other words, each forwarding node can only compute the weight of the edges within its transmission radius.

At each hop, the forwarding node first fixes a sector width bisected by the imaginary line between itself and the target node. Then, it disseminates a short control packet request to send (RTS) containing the location of itself and the final target node to its neighbors. In this case, only nodes which are within the transmission radius can reply to the issued request. After collecting the responses, the forwarding node selects the node with the largest weight. The rationale behind that is to compromise between the two objectives of URLLUC, i.e., reliability and low latency [17]. We draw an exception to this rule, at the final hop, when the target node is within that sector; the packet data is directly forwarded to it even if it does not have the largest edge weight. The process of nodeforwarding-and-selection is repeated until a successful route is established. Fig. 3 illustrates such process. It is essential to mention that all yellow and green dots, in this case, are within the $\mathrm{BER}_{t h}$ from their forwarding node.

The distributed sector-based routing protocol is, therefore, expected to provide the merit of low latency communication. It is more probable to travel less distance because most 


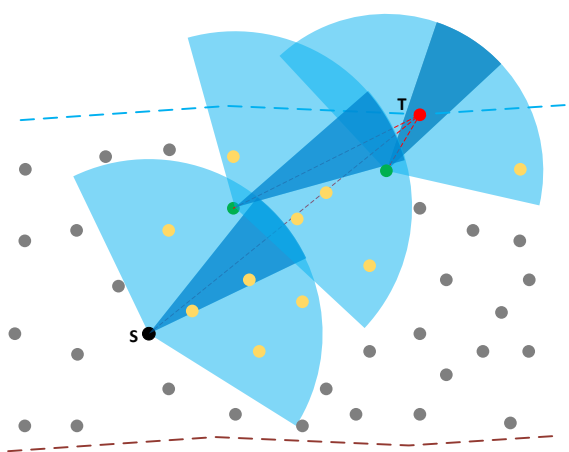

Fig. 4. Path selection process for a sweeping-sector distributed algorithm where the blue sector covers only the allowable transmission area (light blue is the area covered due to sweeping) and the green dots are the forwarding nodes, where the yellow ones are marking the rest of the possible candidates, and the red dashed line is an imaginary line marking how the divergence angle is bisected with respect to the target.

of its candidate nodes are better directed towards the final destination. This routing protocol, however, suffers in term of reliability performance, because of the harsh nature of the fixed distributed sectoring which limits the search area to a low number of candidates (especially for sparse networks and narrowed sectors), so it increments $\mathrm{BER}_{t h}$ more often than the other two algorithms.

\section{Distributed Sweeping-and-Sector-based (DSS) Routing Protocol}

To further overcome the limitations of CRP and DS, we lastly propose the distributed sweeping-and-sector-based (DSS) routing protocol, which is based on tactfully sweeping the forwarding sector bidirectionally (clockwise and anticlockwise) to select a forwarding candidate.

DSS is readily amenable to distributive implementation in practice, and the path selection and data forwarding processes follow the same procedure of the DS protocol. DSS bridges the optical limitations induced innately, i.e., an optical transceiver can transmit with a wider beam-width for a shorter range, and to a longer distance with a narrower beam [18]. Hence by sweeping with a narrow beam, DSS has the opportunity of selecting among an extensive set of vertices with the lower BER links. This is indeed viable since, for the same candidate set as CRP and DS, the probability of error of all edges is less as the transmission occurs with a concentrating beam.

Fig. 3 shows a realization of a selected route using the DSS protocol. Since this algorithm sweeps its sector, this approach covers a larger searching area resulting in a more extensive set of candidates of which may have better error probability. These candidates might, however, be positioned further from the target node, affecting the traveled distance and the number of hops, especially for large sectors.

\section{NuMERICAL RESULTS}

In this section, we assess the performance of all the three routing protocols in terms of end-to-end BER and delay,
TABLE I

SIMULATION PARAMETERS

\begin{tabular}{|l|l|l|l|}
\hline Parameters & Value & Parameters & Value \\
\hline $\mathrm{t}(\lambda)$ Clear ocean $\left[\mathrm{m}^{-1}\right]$ & 0.15 & $\lambda[\mathrm{nm}]$ & 530 \\
$\mathrm{t}(\lambda)$ Coastal ocean $\left[\mathrm{m}^{-1}\right]$ & 0.30 & $R[\mathrm{Mbps}]$ & 1 \\
$\mathrm{t}(\lambda)$ Turbid harbor $\left[\mathrm{m}^{-1}\right]$ & 2.19 & $A\left[\mathrm{~mm}^{2}\right]$ & 0.17 \\
$P_{T x}[W]$ & 2 & $r_{b g}, r_{d c}[\mathrm{~Hz}]$ & $10^{6}$ \\
$\eta_{T}, \eta_{R}, \eta$ & 0.9 & $T[\mathrm{~ns}]$ & 1 \\
\hline
\end{tabular}

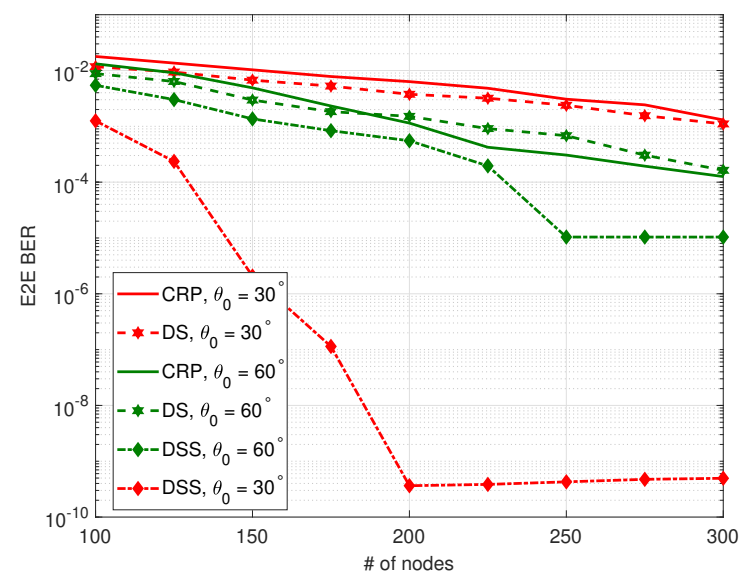

Fig. 5. End-to-end BER performance.

as a function of the number of nodes (i.e., the density of the network). The reason we evaluate the end-to-end BER performance is to ensure the protocols provide a reliable communication link. Additionally, the end-to-end delay evaluation metric is used to examine whether the protocols can support real-time applications or not.

1) Simulations Parameters: We consider a network with a fixed source and a fixed target separated by $145 \mathrm{~m}$ distance over 1000 realizations. The nodes are randomly distributed within an area of $250 \times 250 \mathrm{~m}^{2}$. The parameters listed in Table. I are mainly used throughout the simulations unless stated otherwise.

2) End-to-End Bit Error Rate: Fig. 5 shows the impact of increasing the density of the network on the reliability performance, considering a sector width of $30^{\circ}$ and $60^{\circ}$. The DSS routing protocol has the best performance because it covers a broader search area and has better candidates in terms of error probability, unlike both DS and CRP. Likewise, it performs better with narrowed sectors as all candidates have lower error probability, making it a strong candidate for URLLUC. In principle, both DS and CRP have similar BER performance when it comes to the sector width. Moreover, since the CRP chooses the shortest E2E distance path with disregard of the single weight of the hops, it consequently yields a higher E2E probability of error, making the distributed routing protocols (i.e., DS and DSS) superior.

3) End-to-End Delay: End-to-end delay depends on the total distance traveled and the number of hops in the selected 


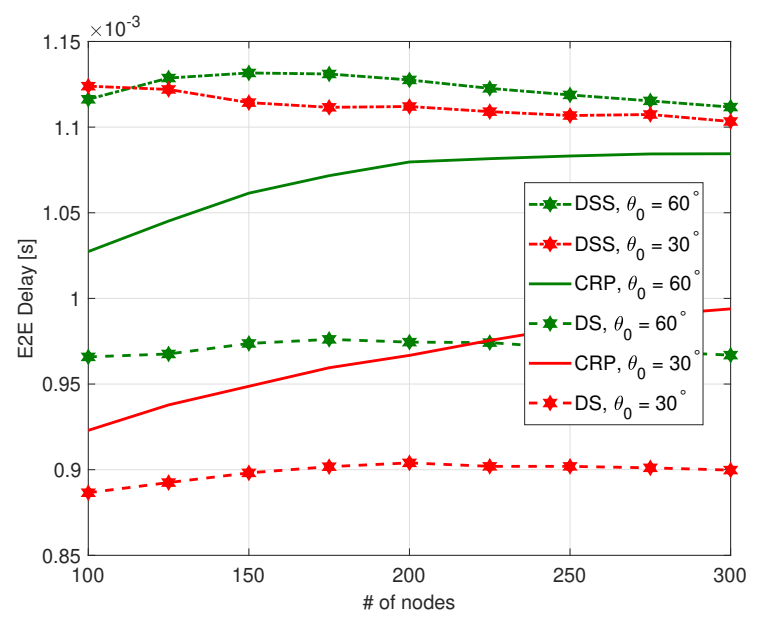

Fig. 6. End-to-end delay performance .

path which is defined as $D_{e 2 e}=K\left(\frac{\zeta}{R}\right)+\frac{d_{S, T}}{c}+(v-1)\left(\frac{\zeta}{R}\right)$, where $K$ is the number of hops in the route, $\zeta$ is the size of the transmitted packet, $d_{S, T}$ is the total traveled distance from source to the target, and $v$ is the number of transmitted packets. Provided that protocols select their transmission path based on the weight of the edges regardless of the number of hops, increasing the density of the network forces the protocols to select a path with a higher number of hops, to have a better end-to-end path cost. Fig. 6 highlights the performance of E2E delay based on the selected paths of each protocol for a 75 bytes message with a packet size of 15 bytes. Equally important, delay performance of the distributed algorithms in Fig. 6 shows a reverse behavior to the reliability metric, leaving us with a trade-off relationship. Fig. 6 shows that all three protocols are meeting the latency requirements of URLLUC. Furthermore, DSS provides high latency, as the source to destination traveled path is typically longer, less directed towards the target, and passes through a large number of forwarding nodes due to sweeping. Similarly, Fig. 6 shows how the proposed DS provides the least delay among the three mainly when operating with narrowed sectors because it can access longer hops and result in a small number of hops. Such observation makes DS the strongest nominee among the three investigated routing protocols for realizing low latency UOWC system. CRP which chooses the shortest E2E distance path shows an unexpected latency performance because sometimes it operates within $B E R_{t h}$ different than the distributed protocols (i.e., DS and DSS), especially in sparse networks, resulting in a longer total distance. Also, given that CRP only considers the total cost (weight) of the path, it eventuates in a considerable number of hops.

\section{CONCLUSIONS}

This paper is one step forward towards realizing ultrareliable low-latency underwater optical communication, by means of efficient routing in underwater optical wireless sensor networks. The paper proposes new routing algorithms which can be implemented in a distributed fashion across UOWSNs.
Numerical results validate the performance and qualification of the proposed routing protocols towards achieving ultra-reliable low-latency underwater optical communication. The proposed distributed sector-based and sweeping-and-sector-based routing protocols exhibit a trade-off in the superiority of their performance to realize URLLUC in the presence of absorption, scattering, and geometrical losses. Finally, the paper elucidates the drawbacks of utilizing a centralized routing protocol in UOWSNs.

\section{REFERENCES}

[1] R. Alghamdi, N. Saeed, H. Dahrouj, T. Y. Al-Naffouri, and M.-S Alouini, "On distributed routing in underwater optical wireless sensor networks," 2018. [Online]. Available: https://arxiv.org/abs/1811.05308

[2] National Oceanic and Atmospheric Administration, "Why should we care about the ocean?" https://oceanservice.noaa.gov/facts/why-careabout-ocean.html, online; accessed 11 September 2018.

[3] S. Arnon and D. Kedar, "Non-line-of-sight underwater optical wireless communication network," J. Opt. Soc. Am. A, vol. 26, no. 3, pp. 530539, Mar. 2009.

[4] L. K. Gkoura, G. D. Roumelas, H. E. Nistazakis, H. G. Sandalidis, A. Vavoulas, A. D. Tsigopoulos, and G. S. Tombras, "Underwater optical wireless communication systems: A concise review," in Turbulence Modelling Approaches, K. Volkov, Ed. Rijeka: IntechOpen, 2017, ch. 9.

[5] N. Saeed, A. Celik, T. Y. Al-Naffouri, and M.-S. Alouini, "Underwater optical wireless communications, networking, and localization: A survey," vol. abs/1803.02442, 2018. [Online]. Available: http://arxiv.org/abs/1803.02442

[6] E. Morgado, I. Mora-Jimenez, J. J. Vinagre, J. Ramos, and A. J. Caamano, "End-to-end average BER in multihop wireless networks over fading channels," IEEE Trans. Wireless Commun., vol. 9, no. 8, pp. 2478-2487, Aug. 2010.

[7] M. Bennis, M. Debbah, and H. V. Poor, "Ultrareliable and low-latency wireless communication: Tail, risk, and scale," Proceedings of the IEEE, vol. 106, no. 10, pp. 1834-1853, Oct 2018.

[8] G. Pocovi, H. Shariatmadari, G. Berardinelli, K. Pedersen, J. Steiner, and Z. Li, "Achieving ultra-reliable low-latency communications: Challenges and envisioned system enhancements," IEEE Network, vol. 32, no. 2, pp. 8-15, Mar. 2018.

[9] C. Tunca, S. Isik, M. Y. Donmez, and C. Ersoy, "Distributed mobile sink routing for wireless sensor networks: A survey," IEEE Communications Surveys Tutorials, vol. 16, no. 2, pp. 877-897, Second 2014.

[10] R. W. L. Coutinho, L. F. M. Vieira, and A. A. F. Loureiro, "DCR: Depth-controlled routing protocol for underwater sensor networks," in IEEE Symposium on Computers and Communications (ISCC), Jul. 2013, pp. 453-458.

[11] J. M. Jornet, M. Stojanovic, and M. Zorzi, "Focused beam routing protocol for underwater acoustic networks," in Proceedings of the Third ACM International Workshop on Underwater Networks. New York, NY, USA: ACM, Sep. 2008, pp. 75-82.

[12] A. Celik, N. Saeed, T. Y. Al-Naffouri, and M.-S. Alouini, "Modeling and performance analysis of multihop underwater optical wireless sensor networks," in IEEE Wireless Commun. and Netw. Conf., (WCNC), Apr. 2018, pp. 1-6.

[13] T. H. Cormen, Introduction to Algorithms. MIT Press, 2008.

[14] S. Arnon, "Underwater optical wireless communication network," Optical Engineering, vol. 49, no. 1, pp. 1-6, Jan. 2010.

[15] A. Vavoulas, H. G. Sandalidis, and D. Varoutas, "Underwater optical wireless networks: A $k$-connectivity analysis," IEEE J. Ocean. Eng., vol. 39, no. 4, pp. 801-809, Oct. 2014.

[16] G. Shakhnarovich, T. Darrell, and P. Indyk, Nearest-Neighbor Methods in Learning and Vision: Theory and Practice. MIT Press, 2005.

[17] M. Haenggi and D. Puccinelli, "Routing in ad hoc networks: A case for long hops," IEEE Commun. Mag., vol. 43, no. 10, pp. 93-101, Oct 2005.

[18] A. Celik, N. Saeed, B. Shihada, T. Al-Naffouri, and M.-S. Alouini, "SectOR: Sector-based opportunistic routing protocol for underwater optical wireless networks," in IEEE Wireless Commun. and Netw. Conf., (WCNC), Apr. 2019, pp. 1-6. 